\section{Rivaroxaban auch im Alltag sicher und effektiv}

In einer Beobachtungsstudie wurden die Effektivität (Schlaganfallprävention) und die Sicherheit (Blutungen) von Rivaroxaban im klinischen Alltag bei Patienten mit nicht valvulärem Vorhofflimmern untersucht.

n XANTUS wurden Patienten mit nicht valvulärem Vorhofflimmern eingeschlossen, bei denen der direkte Faktor-Xa-Inhibitor Rivaroxaban zur Schlaganfallprävention eingesetzt wurde. Als bedeutende klinische Ereignisse wurden schwere Blutungs- und symptomatische thromboembolische Ereignisse (Schlaganfall, TIA, Myokardinfarkt) sowie Tod definiert. Insgesamt wurden 6.784 Patienten in 311 Zentren Europas, Israels und Kanadas untersucht und im Mittel 329 Tage nachverfolgt. Schwere Blutungsereignisse traten bei 128 Patien- ten (2,1 Ereignisse je 100 Patientenjahre) auf, 118 Patienten starben (1,9 Ereignisse je 100 Patientenjahre). Zudem erlitten 43 Patienten (0,7 Ereignisse je 100 Patientenjahre) einen Schlaganfall.

Camm AJ et al. XANTUS: a real-world, prospective, observational study of patients treated with rivaroxaban for stroke prevention in atrial fibrillation. Eur Heart J. 2015; doi: 10.1093/ eurheartj/ ehv466

\section{Kommentar}

Dies ist die erste große prospektive, internationale Beobachtungsstudie, die Daten zur Schlaganfallprävention eines direkten oralen Antikoagulans von „Alltagspatienten“ mit Vorhofflimmern außerhalb klinischer Studien zeigt.

Die klinischen Ergebnisse von Rivaroxaban sind nicht schlechter als die in großen randomisierten Zulassungsstudien und gut vergleichbar mit denen unlängst publizierter Register (z.B. Dresden NOAC Register). Die stetig breiter werdende Datenbasis zur Sicherheit und Effektivität direkter oraler Antikoagulanzien rechtfertigt deren zunehmenden klinischen Einsatz.

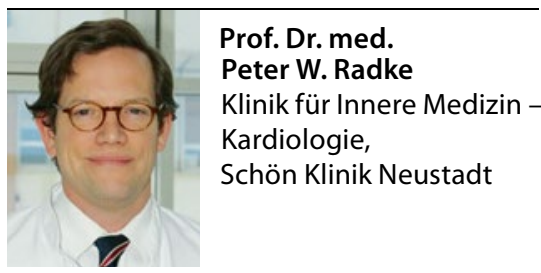

\title{
Mit dem BMI steigt das Krebsrisiko
}

\section{Mit Adipositas werden z. B. Typ-2-Diabetes, Myokardinfarkt, Schlafapnoe und Gonarthrose assoziiert, kaum jedoch maligne Erkrankungen. Welche Rolle spielen adipositasassoziierte Krebskrankheiten?}

A usgewertet wurde eine weltweite Datenbank (GLOBOCAN). Es wurden die Inzidenzraten für Krebskrankheiten basierend auf den Jahren 1982 und 2002 erreichnet. 3,6\% aller neu aufgetretenen Krebsfälle (481.000) waren einem BodyMass-Index (BMI) > $25 \mathrm{~kg} / \mathrm{m}^{2}$ zuzuschreiben. In $13 \%$ der Krebskrankheiten mit starker Assoziation zur Adipositas war ein hoher BMI die Ursache von Karzinomen des Ösophagus, des Kolons, des Rektums, der Gallenblase, der Brust

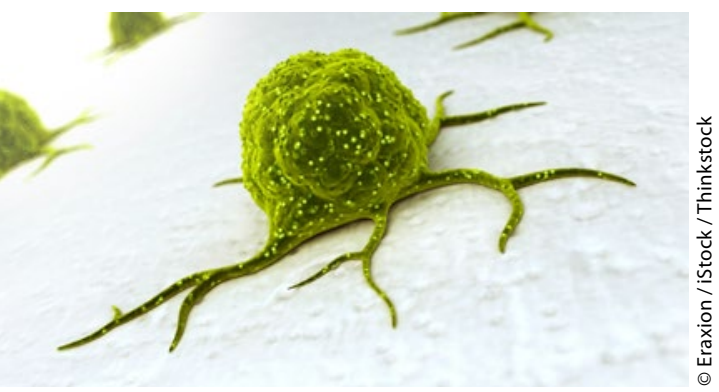

Zur Entwicklung maligner Erkrankungen kann auch eine Adipositas beitragen. (postmenopausal), der Ovarien, der Nieren, des Pankreas und des Uterus. Für die einzelnem Krebsarten wurde die „population attributable fraction“ (PAF) ermittelt, eine Größe, die die Häufigkeit eines hohen BMI und das relative Risiko für eine Krebserkrankung in Betracht zieht. Bei Männern waren $33 \%$ der Ösophagus-, $17 \%$ der Nieren- und $13 \%$ der Kolonkarzinome und bei Frauen 34\% der Uterus- und Ösophagus-, $32 \%$ der Gallenblasen- und 10\% der Mammakarzinome auf einen hohen BMI zurückzuführen. Die Inzidenz der oben erwähnten Krebskrankheiten mit starker Assoziation zur Adipositas war bei Frauen größer als bei Männern.

In Nordamerika waren die PAFs am höchsten, in Asien und in SubsaharaAfrika am niedrigsten. Europa nahm eine Mittelstellung ein, in Osteuropa war das Risiko am höchsten, in Westeuropa an niedrigsten. Wäre der durchschnittliche BMI von 1982 bis 2002 konstant geblieben, hätten ein Viertel aller Krebsfälle verhindert werden können.
Arnold $\mathrm{M}$ et al. Global burden of cancer attributable to high body-mass index in 2012: a population based study. Lancet Oncol. 2015;16:36-46.

\section{Kommentar}

Wenngleich ca. viermal mehr Todesfälle durch Herz-Kreislauf-Krankheiten als durch Krebskrankheiten aufgrund eines hohen BMI entstehen, ist das Studienergebnis basierend auf Inzidenzraten erschreckend. Betrachtet man Krebskrankheiten mit starker Assoziation zu einem hohen BMI, stellt man fest, dass die Adipositas einen Anteil an deren Entstehung von $10-30 \%$ hat. Dieses hohe Krebsrisiko bei zehn Krebsarten aufgrund einer deutlichen Körperfettvermehrung war in diesem Umfang bisher nicht bekannt.

Die Entstehungsmechanismen maligner Erkrankungen sind komplex und kaum geklärt. Adipöse Personen haben hohe Insulin-, IGF-1-, Östrogen- und Leptinspiegel. Diese Faktoren wirken proliferativ, mitogen und z.T. entzündlich, weitere karzinogene Mechanismen sind nachgewiesen.

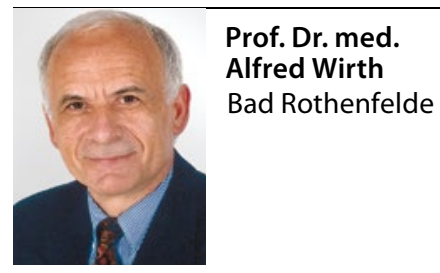

